\title{
An in-depth Study of the Concept of Chirality in Organic and Pharmaceutical Chemistry: Using Crystal Structure Data with 3D Visualization Software
}

\author{
Yi Yang, Jian Xu, Gao Cao* \\ School of Chemistry and Chemical Engineering, Guangdong Pharmaceutical University, Guangdong, CHINA.
}

\begin{abstract}
Background: Chirality is an essential feature of life and a universal phenomenon in nature, chirality in chemistry and biochemistry is closely related to biological, spectroscopic and physical properties. The concept of chirality also has important applications in pharmaceutical chemistry. Objectives: This article aims to introduce how crystal structure data can be used in 3D visualization software to deepen students' understanding of the concept of chirality in organic and pharmaceutical chemistry. Methods: Many crystal data examples are used to introduce chiral compounds and the absolute configuration of chiral factor, such as chiral atoms, axial chirality, planar chirality, spiral chirality and supramolecular chirality, the concrete teaching method is to import the crystal data into the visualization software, rotate it to the appropriate position, then labelled as the absolute configuration. The students were asked to do a set of chiral exercises based on crystal structure data. The effectiveness of this teaching method is judged by classroom test feedback. Results: We successfully established and applied a chiral teaching method based on crystal structure data. The test scores of students using this method were higher than those who had not used it before. Conclusion: This method can help students better understand the concept of chirality, improve the teaching quality and test scores and also lay a foundation for the application of chemistry database in teaching.
\end{abstract}

Key words: Crystal structure data, Chirality, Pharmaceutical chemistry, Educational informatization, Instructional tool, Reform in education.

\section{INTRODUCTION}

Chirality is an important concept in organic and pharmaceutical chemistry. Before the concept of chirality was well understood, some tragedy such as "thalidomide event" led to the most serious event in the history of drug use. Pregnant women who took racemic thalidomide gave birth to more than 12,000 babies with birth defects in the 1950s. Later studies found that the $R$ configuration of thalidomide had a sedative effect, while the $S$ configuration had a strong teratogenic effect on the embryo. ${ }^{1,2}$ In 1992, the Food and Drug Administration in the United States issued "Development of New Stereo isomeric Drugs", requiring manufacturers of racemic new drugs to report on the pharmacologic and toxicological effects of the enantiomers contained in their drugs. In general, an object is called "chiral" if it does not coincide with its image (Figure 1). The chirality of a molecule is usually caused by an asymmetric chiral carbon centre, which is commonly identified by $R / S$ or $D / L$. However, with the deepening of stereoisomerism research and the rapid development of new methods and technologies for molecular structure characterization, it is not appropriate to judge a chiral molecule only by whether it contains chiral carbon. In fact, some compounds that don't have chiral carbon atoms, but do have other chiral factors that don't overlap with their mirror images, such as chiral heteroatoms, axial chirality, planar
Submission Date: 06-06-2020; Revision Date: 25-08-2020; Accepted Date: 07-10-2020

DOI: 10.5530/ijper.54.4.209 Correspondence:

Dr. Gao Cao

School of Chemistry and

Chemical Engineering, Guangdong Pharmaceutical University, Guangdong, CHINA.

Phone no:

+8602039350187

Email id: cg@gdpu.edu.cn

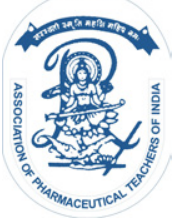

www.ijper.org 


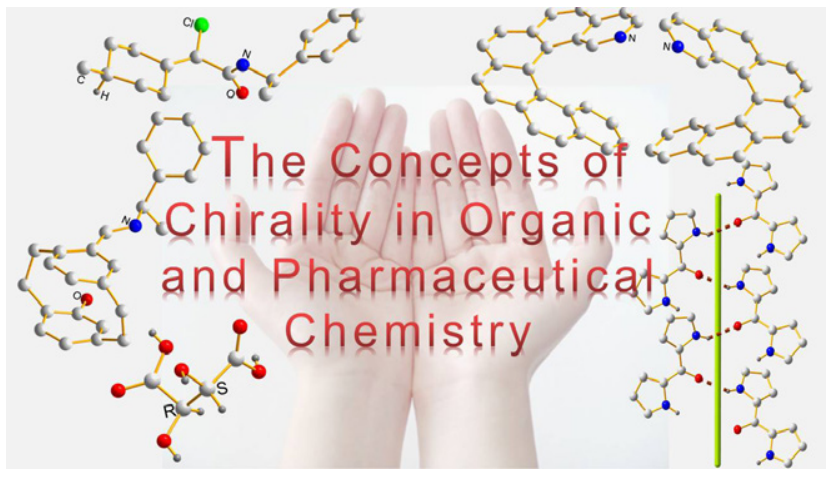

Figure 1: Hands and chirality.

chirality, spiral chirality and supramolecular chirality, these molecules are also chiral molecules. ${ }^{3}$

In the course of teaching organic and pharmaceutical chemistry, the author found chirality to be a difficulty because it was limited by students' spatial imagination. We believe that students can go beyond rigid rote learning and better understand these three-dimensional (3D) structures by cooperating with the explanation in the hands-on process. At first, we asked students to build ball-and-stick models by themselves in class. ${ }^{4}$ However, with the deepening of teaching, these balls and sticks have shortcomings such as insufficient quantity and difficulty in expressing hydrogen bonds when constructing complex structures. To make up for this deficiency, the author used $\mathrm{ChemDraw}^{\circledR}$ and The Cambridge Crystallographic Data Centre (CCDC) software in the computer classroom to have each student draw 3D structures on a computer. International Union of Crystallography (IUCr) defines a file format called CIF for easy exchange of crystallographic information. ${ }^{5}$ Each CIF file contains 3D structure data, two-dimensional structure drawing, chemical formula and compound name, complete literature and other related letters. Researchers upload them to the appropriate database, such as the Cambridge structural database (CSDS), which enables people to study their molecular structure, molecular interaction and cell accumulation, providing a convenient condition for us to apply the crystal data to the teaching field..$^{6-9}$ In this paper, some examples of crystal structure data are given to introduce the chiral compounds and their absolute configurations generated by other chiral factors.

\section{MATERIALS AND METHODS}

The database and software used in this paper: the Cambridge crystal database, Mercury.
The crystal structure data used in this paper are from the CCDC. Their compound names and the codes in the database are given in Table 1.

These data can be obtained free of charge from The CCDC.

We will briefly teach students how to find and use crystal structure data before we start teaching them.

In this process, students need to learn:

1. Search and download crystal structure data in the Cambridge Crystal Database. (https://www.ccdc. cam.ac.uk/structures/)

2. Download and install the Mercury $^{\circledR} 3.6$ Windows Software. (https://www.ccdc.cam.ac.uk/ Community/csd-community/FreeMercury/)

3. Use Mercury ${ }^{\circledR}$ to open the crystal structure data file and rotate the compound to view its structure from multiple angles and view the molecular packing diagram. (Additionally, we can use Diamond ${ }^{\mathbb{B}}$, Material Studio ${ }^{\circledR}$, Power Cell $^{\circledR}, \mathrm{X}$-ceed ${ }^{\circledR}$, etc.)

When our students learn how to find and import crystal structure data in software, we can apply this data to teaching.

To understand the absolute configuration of the chiral compounds is a difficult aspect in teaching process. At present, the nomenclature proposed by Cahn-IngoldPrelog (CIP) is still used internationally to determine the configuration. ${ }^{10}$

In the course of teaching, we ask students to learn each chiral compound in the following way: introduce the configuration determination method of this chiral compound; Draw a 3D model of the substance in the software; Rotate the model to the appropriate position (the position with the least overlap of atoms in the molecule or where the most atoms can be seen); Finally, We led the students to label the configuration of the molecule according to the configuration determination method.

\section{DISCUSSION}

\section{Chiral centers of carbons and heteroatoms}

Taking chiral carbon as an example, the principle is that the four groups attached to the chiral carbon (a, b, c and d) are arranged in order from the large (the priority group) to the small (the last priority group) (assuming the order is $\mathrm{a}>\mathrm{b}>\mathrm{c}>\mathrm{d}$, assigning priorities of substituents are according to CIP sequence rules). The smallest group $\mathrm{d}$ is placed furthest away from the observer's eye and the other three groups are rotated in the direction from large to small. If $\mathrm{a}, \mathrm{b}$ and $\mathrm{c}$ are rotated clockwise, the chiral carbon has an $\mathrm{R}$ configuration; 
Table 1: The CSDS refcodes of the structure shown in the figure in this article.

\begin{tabular}{|c|c|c|}
\hline Figure & Compound name & CSDS (Refcode) \\
\hline Figure 2 & meso-Tartaric acid & TARTAM \\
\hline Figure 2 & $(+)$-Tartaric acid & TARTAL \\
\hline Figure 2 & $D$-Tartaric acid & TARTAC \\
\hline Figure 3 & (-)-(2S)-2-((R)-a-Methylbenzyl)-3,3-diphenyloxaziridine & MBZOCZ20 \\
\hline Figure 3 & 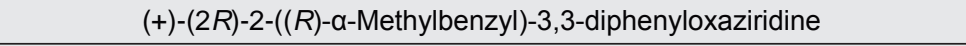 & RRMBXZ \\
\hline Figure 4 & (1S,2S)-(Biphenyl-4-yl)-(2,6-dimethylphenoxy)-(1-phenylethyl)silane & MABCAU \\
\hline Figure 5 & $\begin{array}{c}\left.\left.\text { 2-((S } S_{p}\right)-t-B u t y l-p h e n y l p h o s p h i n o y l\right)-N-((1 S, 2 R)-2-h y d r o x y-1-m e t h y l-2- \\
\text { phenylethyl)acetamide }\end{array}$ & ABOVUJ \\
\hline Figure 7 & $\begin{array}{l}\text { 2-(((+)-2-Oxo-7,7-dimethylbicyclo(2.2.1)hept-1-yl)methylsulfonyl) } \\
\text { amino)-2'-hydroxy-6,6'-dimethyl-1,1'-biphenyl }\end{array}$ & AJALIG \\
\hline Figure 8 & $\begin{array}{l}(R)-(+)-\mathrm{N}-(\alpha-M e t h y l b e n z y l)-(4-m e t h y l c y c l o h e x y l i d e n e) \\
\text { chloroacetamide }\end{array}$ & EABRUU \\
\hline Figure 10 & $\begin{array}{c}\left(R_{\mathrm{p}}, \mathrm{S}_{\mathrm{c}}\right)-4-\text { Hydroxy-15-(1-phenylethyliminomethyl)-(2.2) } \\
\text { paracyclophane }\end{array}$ & AHOREU \\
\hline Figure 11 & rac-Benzo(5,6)phenanthro(3,4-h)isoquinoline & COBPAL \\
\hline Figure 11 & $(P)-(+)$-Benzo(5,6)phenanthro(3,4-h)isoquinoline & BAGLED \\
\hline Figure 12 & 2,2'-Dipyrrolyl ketone & HIXGAW \\
\hline Figure 13 & $L-(+)$-Lactic acid & YILLAG \\
\hline Figure 13 & (S)-2-(2,6-Dioxopiperidin-3-yl)-1H-isoindole-1,3(2H)-dione & ZORBEP \\
\hline Figure 13 & $(R, S)$-1-(t-Butylphosphinyl)-2-(methylphenylphosphinyl)ethane monohydrate & VEVDEF \\
\hline Figure 14 & (R)-2-Amino-2'-dimethylamino-6,6'-dimethyl-1,1'-biphenyl & POVNUK \\
\hline Figure 14 & $(R, R)$-(-)-3-Phenyl-2-ammoniopropanol 4-methylcyclohexylidenecarboxylate & KOVDUU \\
\hline Figure 15 & (S)-(2.2)Paracyclophane-4-carbaldehyde & VECQEA01 \\
\hline Figure 16 & (M)-2,3-Dibromohexahelicene & FILPUN \\
\hline Figure 16 & $(P)$-2,3-Dibromohexahelicene & FILPOH \\
\hline Figure 17 & 2-Amino-4-(t-butyl)-5-(4-chlorobenzyl)thiazol-3-ium bromide & AZEJEV \\
\hline
\end{tabular}

if they are rotated counter clockwise, the chiral carbon has an $S$ configuration.

In class, we'll take tartaric acid (compound A) in Figure 2 as an example. First, we draw it in the software and then we rotate it to a position that is easy to see (i.e. the place with the least overlapping area between atoms and the most visible chemical bonds). After the operation, the students observed the molecule contains two chiral carbons and it has three isomers. Then, the authors led the students to label the chiral atoms, one of them has an $R$ in it and an $S$ in it to form a mesomer, the other two, with two $R$ configurations and two $S$ configurations, are enantiomers of each other. ${ }^{11-14}$

In addition to the common chiral carbon atoms, atoms such as nitrogen, silicon and phosphorus can also become asymmetric centers. ${ }^{15,16}$ Because of the pseudo tetrahedral arrangement of a pair of lone pairs of electrons retained by the nitrogen atom itself and the three substituted groups attached to them, even nitrogen atoms with three different substituents are chiral. However, due to the rapid inversion of nitrogen centers, amines synthesized under most experimental conditions
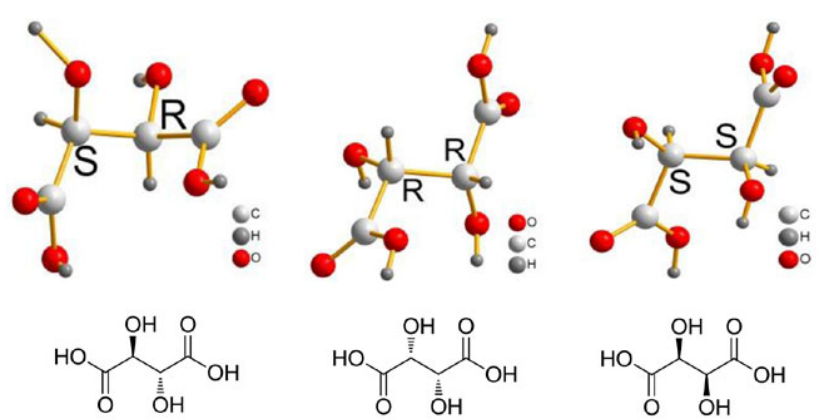<smiles>O=C(O)C(O)[C@@H](O)C(=O)O</smiles>

$\overbrace{\mathrm{O}}^{\mathrm{OH}}$

Compound $(2 R, 3 S)-\mathbf{A} \quad$ Compound $(2 R, 3 R)-\mathbf{A} \quad$ Compound $(2 S, 3 S)-\mathbf{A}$

Figure 2: Carbon chiral centers in tartaric acid.

are racemic and difficult to separate. The enantiomers of chiral nitrogen compounds can be separated only by the formation of quaternary ammonium salts, $\mathrm{N}$-oxides, or other fixed-bonding pathways to block this inversion. A large number of chiral nitrogen compounds in the form of azacyclopropane and azoxycyclopropane were found. For example, the researchers determined the absolute configuration of its nitrogen chiral center by X-ray diffraction (Figure 3). ${ }^{17}$ 


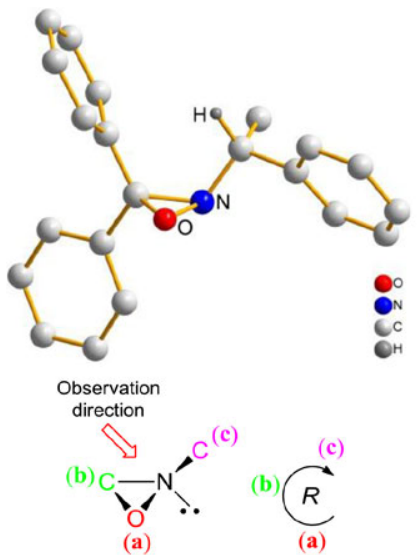

Compound $\left(R_{\mathrm{C}}, R_{\mathrm{N}}\right)-\mathrm{B}$
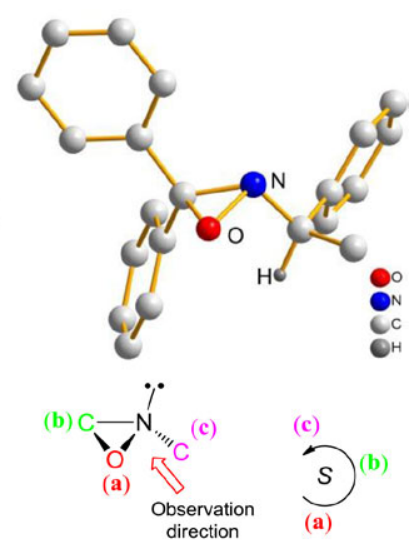

Compound $\left(R_{\mathrm{C}}, S_{\mathrm{N}}\right)-\mathbf{B}$

Figure 3: Nitrogen chiral center in chiral azopropidine. Note: $\mathrm{H}$ atoms that don't affect the configuration have been omitted in the crystal structure, most atoms have been omitted in the structural formula for clarity.
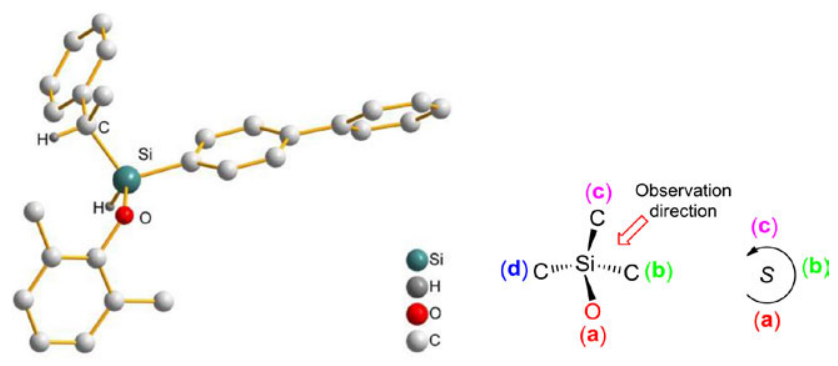

Compound $\left(S_{\mathrm{C}}, S_{\mathrm{Si}}\right)-\mathbf{C}$

Figure 4: Silicon chiral center.

Note: $\mathrm{H}$ atoms that don't affect the configuration have been omitted in the crystal structure, most atoms have been omitted in the structural formula for clarity.

The chiral azoxy heterocyclic propane compound $\mathrm{B}$ was obtained by oxidation of (R)-N-(alpha-methylbenzyl) diphenylmethylene amine. Two non-enantiomers were separated by column chromatography, in which $\left(R_{C}, R_{N}\right):\left(R_{C}, S_{N}\right)=13 \%: 87 \%$. In Figure 3, the absolute configuration of chiral nitrogen in the left and right crystal structure is $R_{N}, S_{N}$, respectively. In addition, the molecule also contains a chiral carbon atom whose absolute configuration is $R_{C}$. The $R / S$ configuration of the chiral nitrogen is named the same as that of the chiral carbon, so students can learn to draw inferences and label them themselves.

Sanji et al. synthesized molecules containing chiral silicon center and through X-ray crystallographic analysis, the stereo structure of the products could be determined, ${ }^{18}$ and the stereochemistry was determined as $\left(S_{\mathrm{C}}, S_{\mathrm{Si}}\right)$-C (Figure 4), it also contains a chiral carbon atom.

Chiral phosphorus and its ligands can efficiently catalyse the reaction and effectively control the stereo selectivity of the pharmaceutical synthesis. Similar to nitrogen atoms, the bonding of phosphorus atoms

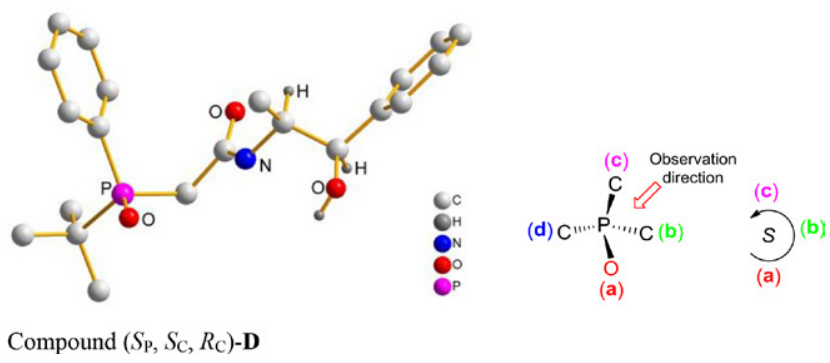

Figure 5: Phosphorus chiral center.

Note: $\mathrm{H}$ atoms that don't affect the configuration have been omitted in the crystal structure, most atoms have been omitted in the structural formula for clarity.
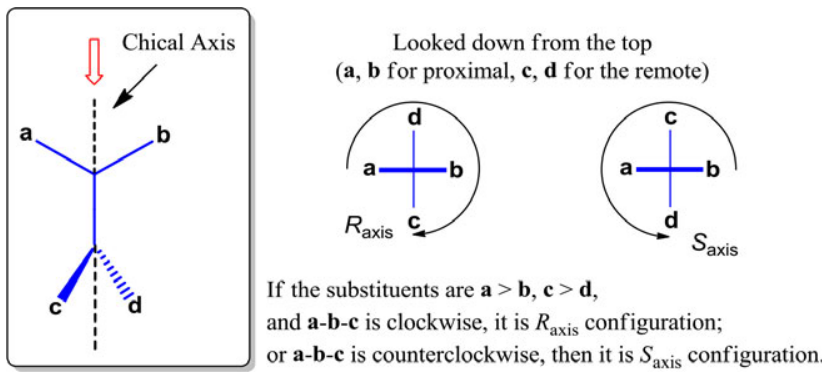

Figure 6: The chiral axis with four substituents and the rules for assigning the configuration of $R_{\text {axis }} / S_{\text {axis }}$.

also has tetrahedral characteristics. Chirality may occur when three or four different groups are attached to phosphorus atoms. And the energy barrier of phosphorus is much larger than that of amine, so it is easier to be split into photo isomers. Previously, Cheng et al. reported a class of aminohydroxy phosphine oxide ligands in ruthenium-catalysed asymmetric transfer hydrogenation reactions. ${ }^{19}$ In this paper, a series of chiral phosphorous compounds, such as this one, are produced in the reaction of $(-)$-menthyl chloroacetate with the anion generated from tert-butylphenylphosphine oxide. The stereo chemical properties of $\left(S_{\mathrm{p}}, S_{\mathrm{C}}, R_{\mathrm{C}}\right)$-D can be determined by single crystal X-ray crystallography (Figure 5).

\section{Axial chirality}

In general, the chirality of organic molecules is due to the chiral center, but in the case of axial chirality, there is no chiral center in the molecule but a chiral axis. The arrangement of multiple groups around the chiral axis may make the molecules unable to coincide with their mirror image, that is, a, b, c, d are not on the same plane and $a \neq b, c \neq d$, thus reflecting the axial chirality (Figure 6).

Axial chirality can also be marked by the $R_{\text {axis }} / S_{\text {axis }}$ configuration. In Figure 6, the rule is: Looking from the top to bottom, the groups seen first are near, denoted by thick solid lines, the groups seen later are 
far, denoted by fine solid lines and then from the large groups near to the small groups at the end and then to the large groups at the far end. The $R_{\text {axis }}$ configuration and the $S_{\text {axis }}$ configuration (counter clockwise) are the smallest atoms or groups placed farthest away from the observer, regardless of the small distal groups, similar to the $R_{\text {axis }} / S_{\text {axis }}$ configuration of the chiral carbon center. So in some cases the observer can approximate the axis (a line) as a point and if the students understand this, the problem of the chiral axis can be readily solved.

The common chiral molecules include allene, spiro, biphenyl and double bond outside the ring. Compound $\mathrm{E}$ is a biphenyl chiral axial compound, which has four substituent groups $\left(-\mathrm{OH},-\mathrm{CH}_{3},-\mathrm{N}-,-\mathrm{CH}_{3}\right)$ in the ortho position of the biphenyl system. Liang et al. determined its absolute configuration by $\mathrm{X}$ single crystal diffraction technique, ${ }^{20}$ its molecular formula and crystal structure are shown in Figure 7. Crystal structure from the top down, a-b-c rotation is in the counter clockwise direction, so the absolute configuration of the chiral axis compound $\mathrm{E}$ is $S_{\text {axis }}$.

Compound $\mathrm{F}$ is an exocyclic double bonded axially chiral compound. ${ }^{21}$ The crystal structure of $\mathrm{F}$ is shown in Figure 8 . The chiral axis occurs when two substituents on the end of the double bond and two substituents on the other side of the ring are distinct from each other. According to the method of judging the chiral axis $R / S$ configuration in Figure 6 (see the whole double bond
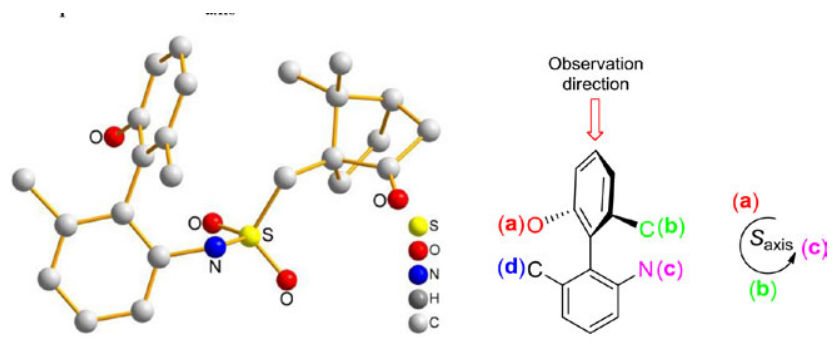

Compound $\left(S_{\text {axis }}\right)-\mathbf{E}$

Figure 7: The chiral axis of biphenyl compounds.

Note: $\mathrm{H}$ atoms that don't affect the configuration have been omitted in the crystal structure, some atoms have been omitted in the structural formula for clarity.
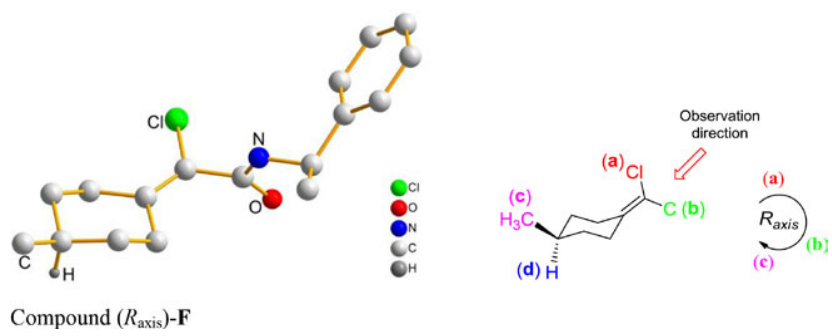

Figure 8: The chiral axis of exocyclic double bond compound. Note: $\mathrm{H}$ atoms that don't affect the configuration have been omitted in the crystal structure, some atoms have been omitted in the structural formula for clarity. and ring as chiral axis), the absolute configuration of the compound is $R_{\text {axis }}$.

\section{Planar chirality}

When substituents exist in a plane or ring made up of atoms and another space bridge exists above or below the plane/ring, plane chirality may generate. The plane chirality is produced by the symmetric breaking of the plane. Typical chiral plane compounds are bridged aromatic compounds and bridged olefin structural compounds. As shown in Figure 9, bridged aromatic compounds are the most common chiral plane compounds. ${ }^{22-24}$

Like axial chirality, planar chirality can also be labelled with an $R_{\text {plane }} / S_{\text {plane }}$ configuration. With an example from the CSDS database, the author introduces a method for labeling planar absolute configurations of p-aryl compounds in class.

The absolute configuration of planar chiral compound $G$ is marked in Figure $10 .{ }^{25}$ Compound $G$ had two chiral planes, namely, the benzene ring plane with phenylethylaminomethyl and the hydroxyl substituted benzene ring. The first step is to determine a labelled atom $\mathrm{P}$, which does not belong to the chiral plane but is connected to the atom a in the chiral plane. When there are two labelled atoms to choose from, the atom that is connected to the high-priority substituent group with a short path is selected as the labelled atom P. In the second step, a path is created along the chemical
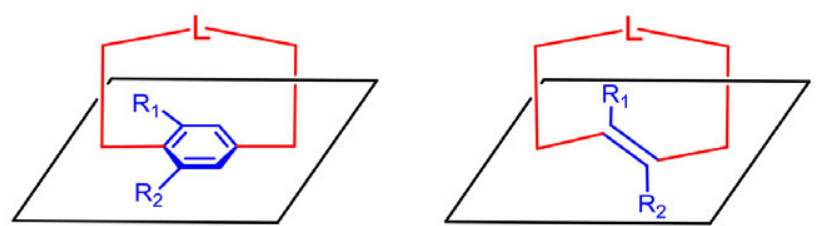

Figure 9: Two kinds of chiral planes.

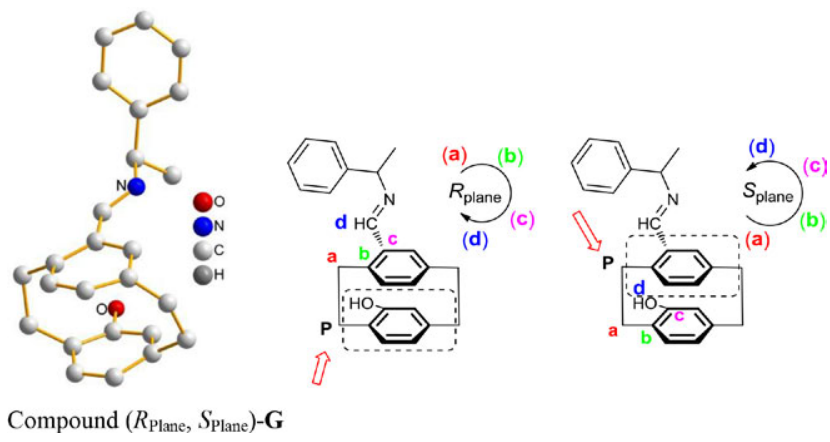

Figure 10: The chiral plane and the rules for assign in the absolute configuration.

Note: $\mathrm{H}$ atoms that don't affect the configuration have been omitted in the crystal structure, some atoms have been omitted in the structural formula for clarity. 
bond from one atom to the next, starting with the atom connected to the labelled atom $\mathrm{P}$. The next atom is always selected with higher priority, until the path can show the cis-inverse trend loop. Finally, if the path created is clockwise, the chiral plane is $R_{\text {plane }}$ and the anticlockwise direction is $S_{\text {plane }}$. In compound $G$, the first chiral plane is $R_{\text {plane }}$ and the second is $S_{\text {plane }}$.

\section{Helical chirality}

The most familiar spiral structure is the double helical structure of DNA. Due to its 5'-3' directionality, a DNA sequence is distinct from a mirrored sequence arranged in reverse nucleotide-order and is therefore chiral. ${ }^{26,27}$ Helical chirality is a special case of chirality in which the molecule is shaped like a right-handed or left-handed screw or spiral escalator. When viewed from above the rotation axis, the spirals seen are clockwise labelled $P$ (right helix) and counter-clockwise labelled $M$ (left helix). The structure of DNA is difficult to draw, so we use a simpler example in class. The CSDS database also contains many helical chiral crystal structures, such as the pairs of enantiomers (Figure 11). ${ }^{28,29}$ From the top down, the structure to the left of the mirror (the dotted line) spirals anticlockwise, so its absolute configuration is $M$; The structure on the right of the mirror, seen from above, spirals clockwise, so its absolute configuration is $P$.

\section{Supramolecular chirality}

Supramolecular refers to aggregates composed of two or more molecules that do not form covalent bonds. Some drugs work in the body for this reason, so further exploration of chiral molecules at the supramolecular
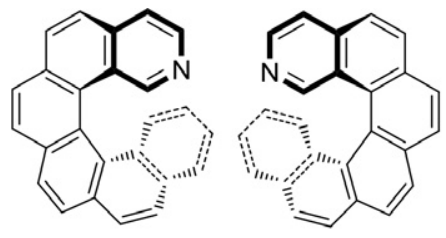

Compound $\mathbf{H}$

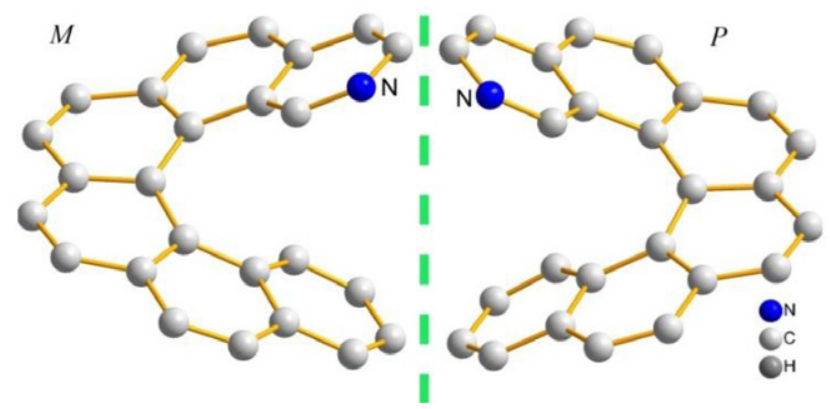

Figure 11: The $M / P$-helix of compound $\mathrm{H}$.

Note: $\mathrm{H}$ atoms bonded to benzene ring have been omitted in the crystal structure. level could help to develop new drugs and materials. A typical example is the secondary structures of proteins, which can exhibit various conformations with different supramolecular chirality. Supramolecules can also construct high-level chiral stereo structures through other self-assembly driving forces such as hydrogen bonding, electrostatic interaction, hydrophobic effect, aromatic ring $\pi-\pi$ stacking and metal ligand interaction, which can make molecules without chirality display chiral signals under certain conditions. In the case where continuous hydrogen bonds in the crystal link the molecule into a helix, the left helix is labelled $M$ and the right helix is labelled P. ${ }^{30,31}$ These theoretical terms seem quite obscure to the students, so the author found a very simple structure to show them the process of generating chirality.

During the synthesis of $\mathrm{C}_{2}$-symmetric porphyrins, Norsten et al. prepared 2,2'-dipyrrolyl ketone I. ${ }^{32}$ This simple molecule contains a high degree of encoded molecular recognition functionality. It contains three hydrogen bond sites, the carbonyl $(\mathrm{C}=\mathrm{O})$ and two amine $(\mathrm{N}-\mathrm{H})$ donors. It was then demonstrated that the single molecule 2,2'-dipyrrolidone passes through the intermolecular $\mathrm{N}-\mathrm{H} \ldots \mathrm{O}=\mathrm{C}$ binds together and selfassembles into a supramolecular helices (Figure 12).

After drawing a single molecule, the students used the replication function to place multiple single molecules on the canvas and then connected the hydrogen bonds with dotted lines, rotating the whole structure and observing it as they went. Now, some of the smartest students have seen that it's chiral and if you add an axis

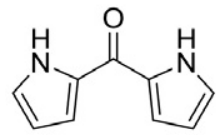

Compound I

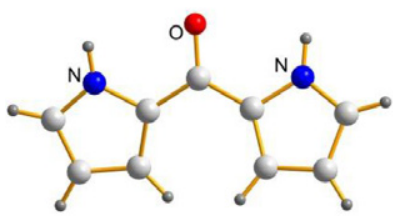

(a)

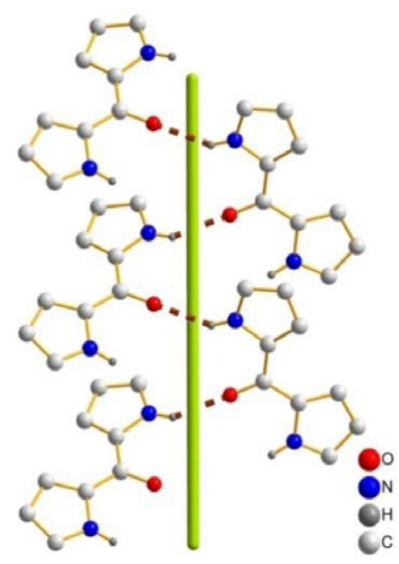

(b)

Figure 12: (a) The single-molecule of compound I; (b) View of hydrogen bond interactions between adjacent molecules in the $M$-helix.

Note: $\mathrm{H}$ atoms that don't affect the configuration have been omitted in the crystal structure, the dotted line indicates the hydrogen bond. 
to it, all the students will suddenly be enlightened that this is the creation of supramolecular chirality.

\section{Test, answer and explanation of chirality}

We also designed a test, in which the examples are similar to those used in class, but different, so that students can flexibly use their knowledge.

Question 1. Mark the chiral centers in the following molecule and determine their configurations. Software can be used to help solve the problem. The crystal structure data is encoded as YILLAG, ${ }^{33}$ ZORBEP, $^{34}$ VEVDEF ${ }^{35}$ respectively.

In Figure 13(a), the four groups linked on the chiral carbon are ordered by CIP sequence rules as the carboxyl $>$ hydroxyl $>$ methyl $>$ hydrogen atoms. We put the hydrogen atoms in the farthest place from the observer and the remaining three groups from the observer direction are counterclockwise from large to small, so this chiral carbon atom is $S$ configuration.

In Figure 13(b), known as a thalidomide and it omits a hydrogen atom attached to its chiral carbon, an omission that often occurs in crystal structure data. According to CIP sequence, nitrogen atom $>$ carbonyl $>$ methylene, three groups are arranged counterclockwise, which can be judged as $S$ configuration.

In Figure 13(c), there are two chiral phosphates, which are ordered by CIP sequence rules mentioned above by oxygen atom $>$ phenyl $>$ tert-butyl $>$ carbon chain, sorted clockwise and $\mathrm{R}$ configuration; and oxygen

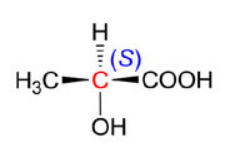

(a)

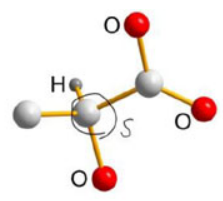

(b)
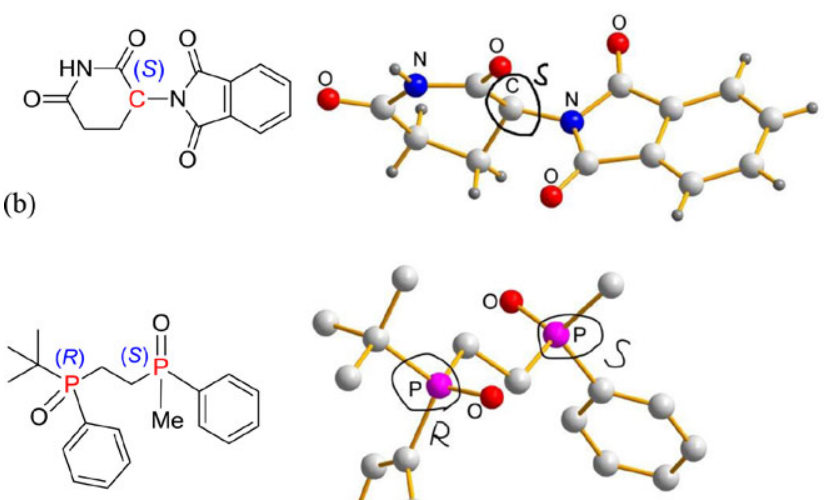

(c)

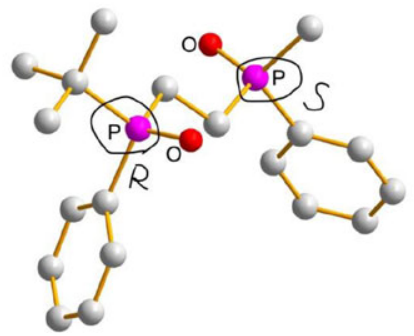

Figure 13: Attached drawing to question 1 (with answers). atom $>$ phenyl $>$ carbon chain $>$ methyl, sorted counterclockwise, is $S$ configuration.

These three configuration problems are not difficult to judge. Most students can tell them without the help of crystal structure data and they only need to be familiar with CIP sequence rules to do them well.

Question 2. Mark the chiral axis of the following compounds and determine their configurations. The crystal structure data is encoded as POVNUK, ${ }^{36}$ KOVDUU. ${ }^{37}$

In Figure 14(a), we see a biphenyl compound, which looks at the bond between two benzene rings as a chiral axis. Using CIP sequence rules to arrange the order, we know that the amino > methyl group on the benzene ring at the top and the dimethyl amino $>$ methyl group on the benzene ring at the bottom. The observer looks down from the top and the a-b-c is arranged clockwise, that is, the $R_{\text {axis }}$ configuration.

In Figure 14(b), we view the six-element ring and the double bond as the chiral axis. Using CIP sequence rules to arrange the order, the carboxyl $>$ hydrogen atom, the methyl $>$ hydrogen atom, the observer looks from the upper right corner to the lower left corner, the a-b-c is arranged clockwise, that is, the $R_{\text {axis }}$ configuration.

The difficulty with these two problems is to find the chiral axis. Students need to remember that the common chiral axes are alkenes, spirals, biphenyls and outer double bonds.

Question 3. Determine the configuration of this compound and label $\mathrm{P}$ and $\mathrm{a}, \mathrm{b}, \mathrm{c}$, d. The crystal structure data is encoded as VECQEA01. ${ }^{38}$
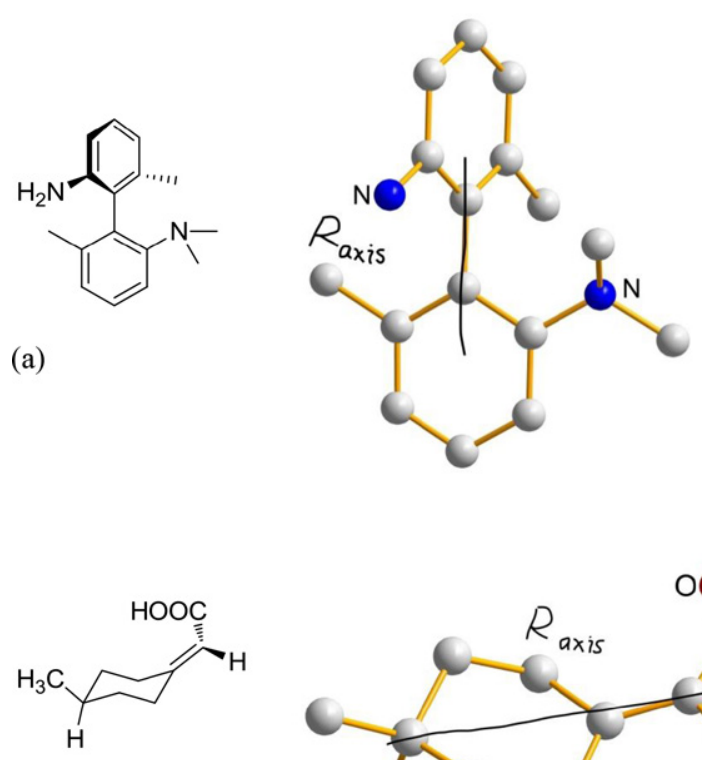

(b)

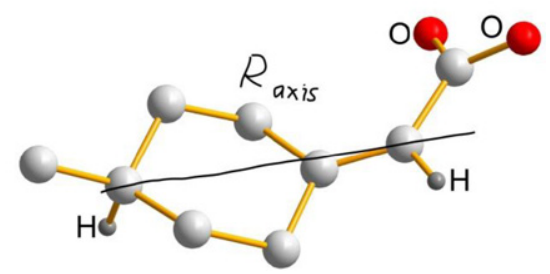

Figure 14: Attached drawing to question 2 (with answers). 
Figure 15 shows a chiral plane, the aldehyde substituted benzene ring plane. We identify a labeled atom $\mathrm{P}$ (which satisfies two conditions that are not on the chiral plane, but are connected to atom a on the chiral plane). Let's label the atom on the surface of $\mathrm{P}$ as $\mathrm{a}$ and the atom on the surface of $\mathrm{a}$ as $\mathrm{b}$ and then the carbon on the two benzene rings that are connected to b, let's choose the carbon that has a shorter path to the substituent as $\mathrm{c}$ and the carbon on the aldehyde group as d. If you look at the plane from P, a-b-c-d is counterclockwise, that is, $S_{\text {plane }}$.

Question 4. Determine the configuration of the following molecules. The crystal structure data is encoded as FILPUN, FILPOH. ${ }^{39}$

From the top down, the structure to the left of the mirror (the dotted line) spirals anticlockwise, so its absolute configuration is $M$; The structure on the right of the mirror, seen from above, spirals clockwise, so its absolute configuration is $P$. (Figure 16)

In our after-class discussion with the students, we found that the spiral structure was the most difficult chiral structure for them to understand. Some students think that a helix can be flipped to form symmetry, but by looking at the crystal structure data we can see that no matter how we rotate the compound, it can never be changed from counterclockwise to clockwise, or from clockwise to counterclockwise.

Question 5. Draw the hydrogen bonds in the molecular packing diagram below to form supramolecular chirality. The crystal structure data is encoded as AZEJEV. ${ }^{40}$

Bromine and hydrogen atoms can form hydrogen bonds together, when the $\mathrm{N}-\mathrm{H}$...Br bonds are joined by dotted lines, we can see visually in the software that they form a counter clockwise spiral, which is the $M$ configuration. (Figure 17)

This problem is also difficult for students to understand. It is hard to imagine the direction of the spiral when you see this problem on paper, but once you introduce

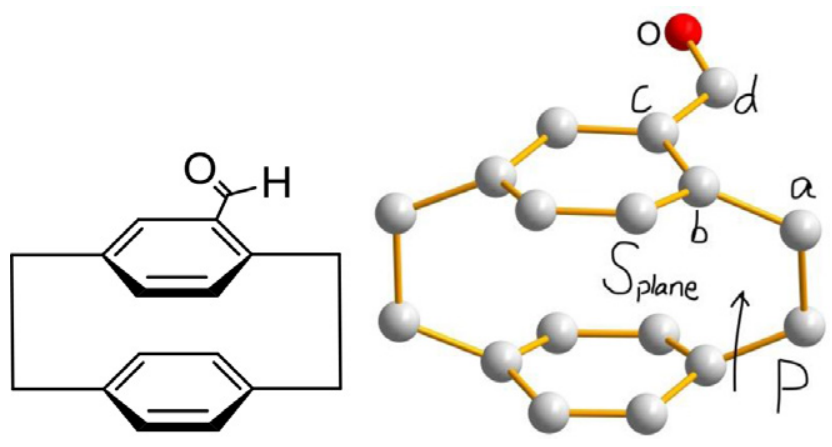

Figure 15: Attached drawing to question 3 (with answers).
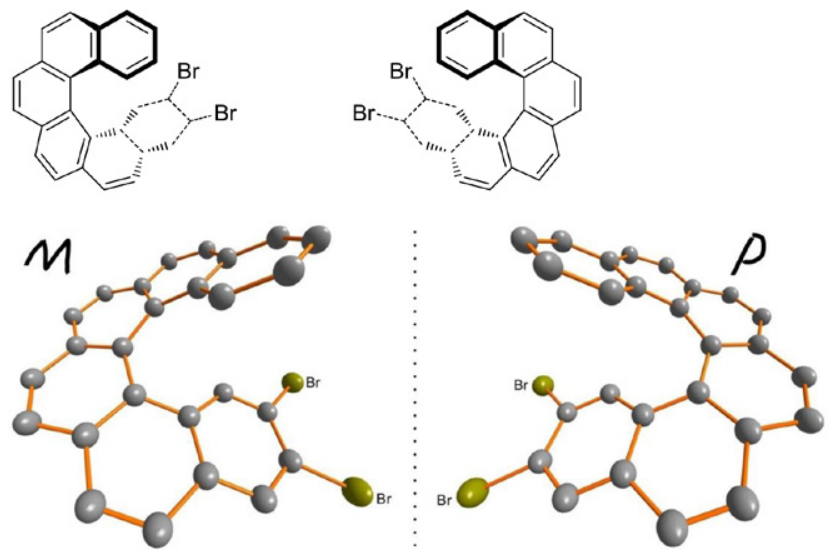

Figure 16: Attached drawing to question 4 (with answers).
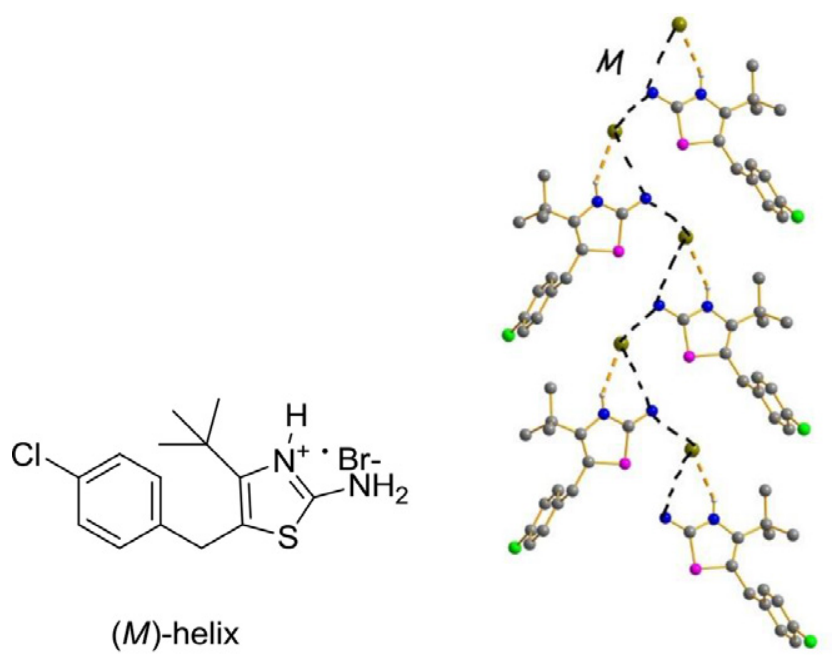

(M)-helix

Figure 17: Attached drawing to question 5 (with answers).

the crystal structure into the visualization software, the problem is easy solved.

\section{Data Analysis}

Since 2016, we have used crystal structure data to teach the concept of chirality. In order to observe the learning benefits brought by this method to students, the scores of the classroom test in organic chemistry of sophomores were collected as reference data in recent years (Figure 18). The statistics include the scores of about 500 students each year from majors including pharmacy, chemical engineering and technology, Chinese materia medica and applied chemistry. (Figure 19) We can clearly see that after 2016 , the failure rate dropped from $20 \%$ to $8 \%$ and the good rate also increased significantly, from $14 \%$ to $27 \%$. In view of the importance and stability of this change, we can think that this teaching method can solve the difficult problems in the teaching, so that students 


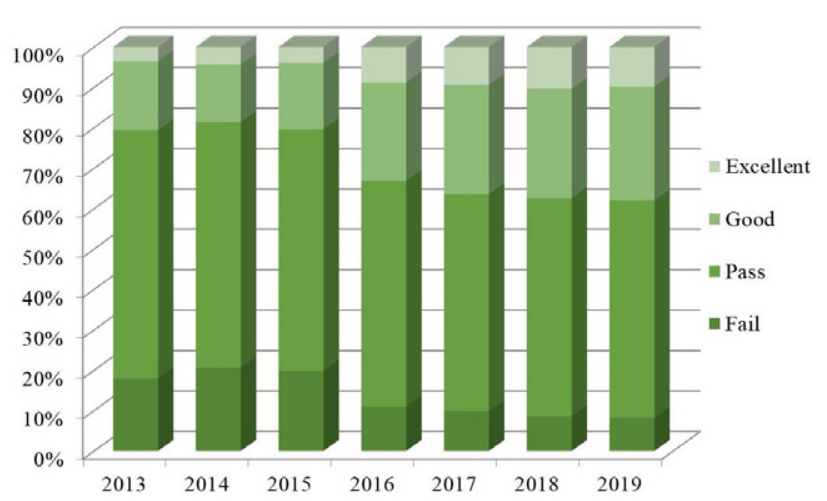

Figure 18: From 2013 to 2019, the cumulative bar chart of the percentage of test scores of our sophomore students.

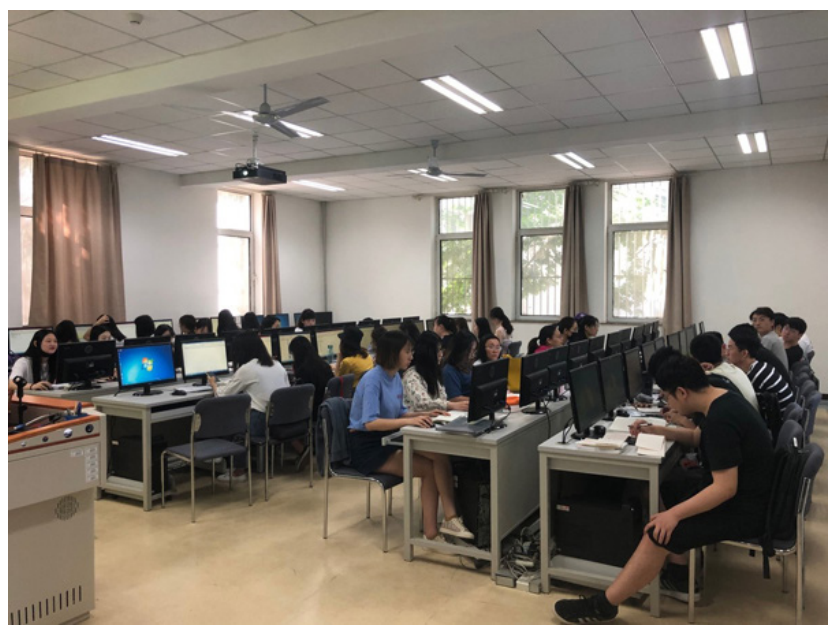

Figure 19: The students have their lessons in the computer classroom.

have a deeper understanding of the concept of chirality and improve their performance.

Our teaching methods are constantly improving. For example, our curriculum also includes the use of ChemDraw $^{\circledR}$ to draw structures, so this data only represents the study status of our students in recent years. We welcome colleagues to communicate with us and share relevant experience.

\section{CONCLUSION}

Since chirality is closely related to drug efficacy, we should pay more attention to the study of this concept. In this paper, chiral compounds and their absolute configurations are in-depth introduced one by one. Our classroom teaching is basically carried out in the order of "explain concepts-import data-rotate molecules-determine configurations" as mentioned above, which can also provide students with some background knowledge of sample compounds so that they can appreciate the mystery of chirality. Since we applied this teaching method to practice in 2016, students' understanding of the concept of chirality has deepened significantly.

From our point of view, the teaching of chirality with crystal structure data has several advantages. First, students can see chiral compounds from multiple angles and have an intuitive feeling of chirality. Second, compared with students passively listening to teachers indoctrinate knowledge, this kind of interesting interactive teaching can stimulate students' interest and enthusiasm to participate and complete learning tasks in the participation. Third, the students learned to use the software of the chemistry major, which laid the foundation for their future study of several chemistry courses and prepared them to participate in the research later.

In the future teaching process, we will search and use more novel auxiliary means. Other researchers have provided us with many options, such as the cholesterol liquid crystals method, the crystalline sponge method and the paper helicopter method, ${ }^{41-43}$ as well as other more intuitive methods to enable students to learn to understand the concept of chirality. We believe that diversified teaching methods can improve students' interest and memory of knowledge.

\section{ACKNOWLEDGEMENT}

The authors would like to thank the Higher Education Reform Project of Guangdong Province and the Provincial Experimental Teaching Demonstration Center of Pharmaceutical and Chemical Industry of Guangdong Pharmaceutical University for its funding.

\section{CONFLICT OF INTEREST}

The authors individually declare that each has no conflict of interest.

\section{ABBREVIATIONS}

3D: Three-Dimensional; CCDC: The Cambridge Crystallographic Data Centre; IUCr: International Union of Crystallography; CIF: Crystallographic Information File; CSDS: The Cambridge structural database; DOI: Digital Object Identifier; CIP: Cahn-Ingold-Prelog; DNA: Deoxyribonucleic Acid.

\section{REFERENCES}

1. Singh N, Sharma L. Enantioseparation of $D$ - and $L$-isomers of chiral drugs for improving their bioavailability: Some techniques including micellization with gemini surfactants. Indian J Pharm Educ Res. 2018;52(3):334-41. 
2. Vargesson N. Thalidomide-induced teratogenesis: History and mechanisms. Birth Defects Res C Embryo Today. 2015;105(2):140-56.

3. Lees P, Hunter RP, Reeves PT, Toutain PL. Pharmacokinetics and pharmacodynamics of stereoisomeric drugs with particular reference to bioequivalence determination. J Vet Pharmacol Ther. 2012;35(Suppl.1):17-29.

4. You Q, Lin G. Chiral drugs: Research and application. Beijing: Chemical Industry Press. 2004.

5. Brown ID, Mcmahon B. CIF: The computer language of crystallography. Acta Crystallogr. 2010;58(3-1):317-24.

6. Battle GM, Ferrence GM, Allen FH. Applications of the Cambridge structural database in chemical education. J Chem Educ. 2010;43(5):1208-23.

7. Battle GM, Allen FH, Ferrence GM. Teaching three-dimensional structural chemistry using crystal structure databases. 3 . The Cambridge structural database system: information content and access software in educational applications. J Chem Educ. 2011;88(7):886-90.

8. Battle GM, Allen FH. Learning about intermolecular interactions from the Cambridge structural database. J Chem Educ. 2012;89(1):38-44.

9. Cao G. Application of Cambridge structural database in stereochemistry teaching. University Chemistry. 2012;27(1):48-50.

10. Cahn RS, Ingold C, Prelog V. Specification of molecular chirality. Angew Chem Int Ed. 1966;5(4):385-415.

11. Okaya Y, Stemple NT, Kay MI. Refinement of the structure of $D$-tartaric acid by X-ray and neutron diffraction. Acta Crystallogr. 1966;21(2):237-43.

12. Bootsma GA, Schoone JC. Crystal structures of mesotartaric acid. Acta Crystallogr. 1967;22(4):522-32.

13. Albertsson J, Oskarsson $\AA$, Ståhl K. A liquid-helium cryostat for collection of three-dimensional $\mathrm{X}$-ray intensity data down to $20 \mathrm{~K}$. The crystal structure of $D$ (+)-tartaric acid at 35, 105, 160 and $295 \mathrm{~K}$. J Appl Crystallogr. 1979;12(6):53744.

14. Xuan J, Feng Y. Enantiomeric tartaric acid production using cis-epoxysuccinate hydrolase: History and perspectives. Molecules. 2019;24(5):903-13.

15. Feng HX, Tan R, Liu YK. An efficient one-pot approach to the construction of chiral nitrogen-containing heterocycles under mild conditions. Org Lett. 2015;17(15):3794-7.

16. Eckert PK, Golz C, Degen P, Werner C, Rehage H, Strohmann C. Exploring the synthesis of a new group of chiral ammonium salts with specific configurations at the stereogenic nitrogen centers. Chem: Eur J. 2014;20(12):3268-72.

17. Forni A, Garuti G, Moretti I, Torre G, Andreetti GD, Bocelli G, et al. Absolute configuration at the chiral nitrogen atom in optically active oxaziridines. $X$-ray structure, physical properties and thermal epimerization of $(-)-(2 S)$ -

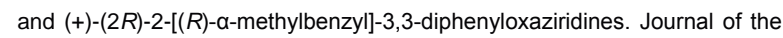
Chemical Society, Perkin Transactions 2. 1978;(5):401-5.

18. Sanji T, Fujiyama H, Yoshida K, Sakurai $H$. Diastereoselective addition of alcohol to diastereotopic silylenes. J Am Chem Soc. 2003;125(11):3216-7.

19. Cheng X, Horton PN, Hursthouse MB, Hii KKM. Aminohydroxy phosphine oxide ligands in ruthenium-catalysed asymmetric transfer hydrogenation reactions. Tetrahedron: Asymmetry. 2004;15(14):2241-6.

20. Liang $\mathrm{Y}, \mathrm{Gao} \mathrm{S}$, Wan $\mathrm{H}$, Wang J, Chen $\mathrm{H}$, Zheng Z, et al. Syntheses and resolutions of new chiral biphenyl backbones: 2-amino-2'-hydroxy-6,6'dimethyl-1,1'-biphenyl and 2-amino-2'-hydroxy-4,4', 6,6'-tetramethyl-1,1'biphenyl. Tetrahedron: Asymmetry. 2003;14(10):1267-73.

21. Topolski M, Duraisamy M, Rachon J, Gawronski J, Gawronska K, Goedken V, et al. Chiral carbenoids: Their formation and reactions. J Org Chem. 1993;58(3):546-55.

22. Stetsovych $\mathrm{O}$, Švec $M$, Vacek $J$, Chocholoušová JV, Jančařík $A$, Rybáček J, et al. From helical to planar chirality by on-surface chemistry. Nat Chem. 2017;9(3):213-8.

23. Morisaki Y, Sawada R, Gon M, Chujo Y. New Types of Planar Chiral [2.2] Paracyclophanes and Construction of One-Handed Double Helices. Chem: Asian J. 2016;11(18):2524-7.
24. Zhou Z, Cao G. Teaching method for determination of absolute configurations of planar chiral compounds. Chinese Journal of Chemistry Education. 2015;36(10):19-21.

25. Rozenberg VI, Antonov DY, Sergeeva EV, Vorontsov EV, Starikova ZA, Fedyanin IV, et al. Enantiomerically pure (R)- and (S)-15-hydroxy [2.2] paracyclophane-4-carbaldehyde (iso-FHPC): A novel parent compound for planar chiral ligands. Eur J Org Chem. 2003;11:2056-61.

26. Deveson IW, Madala BS, Blackburn J, Barker C, Wong T, Barton KM, et al. Chiral DNA sequences as commutable controls for clinical genomics. Nat Commun. 2019;10(1):1342.

27. McDermott ML, Vanselous $\mathrm{H}$, Corcelli SA, Petersen PB. DNA's chiral spine of hydration. ACS Cent Sci. 2017;3(7):708-14.

28. Míšek J, Teplý F, Stará IG, Tichý M, Šaman D, Císařová I, et al. A straightforward route to helically chiral $\mathrm{N}$-heteroaromatic compounds: practical synthesis of racemic 1,14-diaza [5] helicene and optically pure 1-and 2-aza [6] helicenes. Angew Chem Int Ed. 2008;47(17):3188-91.

29. Matsumoto A, Yonemitsu K, Ozaki H, Míšek J, Starý I, Stará IG, et al. Reversal of the sense of enantioselectivity between 1- and 2-aza [6] helicenes used as chiral inducers of asymmetric autocatalysis. Org Biomol Chem. 2017;15(6):1321-4.

30. Liu M, Zhang L, Wang T. Supramolecular chirality in self-assembled systems. Chem Rev. 2015;115(15):7304-97.

31. Kurata M, Yoshizawa A. The formation of a chiral supramolecular structure acting as a template for chirality transfer. Chem Commun. 2020;56(59):8289-92.

32. Norsten TB, McDonald R, Branda NR. The self-assembly and spontaneous resolution of a hydrogen-bonded helix. Chem Commun. 1999;(8):719-20.

33. Schouten A, Kanters JA, Krieken JV. Low temperature crystal structure and molecular conformation of $L-(+)$-lactic acid. J Mol Struct. 1994;323:165-8.

34. Maeno M, Tokunaga E, Yamamoto T, Suzuki T, Ogino Y, Ito E, et al. Selfdisproportionation of enantiomers of thalidomide and its fluorinated analogue via gravity-driven achiral chromatography: Mechanistic rationale and implications. Chem Sci. 2015;6(2):1043-8.

35. Pietrusiewicz KM, Zabłocka M, Wieczorek W. Determination of the absolute configurations of 1,2-diphosphinylethanes and 1,2-diphosphinylethanes possessing two nonequivalent asymmetric phosphorus centres. Crystal structure of $(R, S)$-1-(tert-butylphenylphosphinyl)-2-(methyl-phenylphosphinyl) ethane. Phosphorus, Sulfur Silicon Relat Elem. 1989;42(3-4):183-90.

36. Zi G, Liu X, Xiang L, Song H. Synthesis of group 4 metal amides with new chiral biaryldiamine-based ligands and their use as catalysts for asymmetric hydroamination/cyclization. Organometallics. 2009;28(4):1127-37.

37. Walborsky HM, Goedken VL, Gawronski JK. The absolute configuration of (R)-(-)-(4-methylcyclohexylidene) acetic acid. J Org Chem. 1992;57(1):410-1.

38. Negru M, Schollmeyer D, Kunz $H$. Enantioselective strecker reaction catalyzed by an organocatalyst lacking a hydrogen-bond-donor function. Angew Chem Int Ed. 2007;46(48):9339-41.

39. Biet T, Fihey A, Cauchy T, Vanthuyne N, Roussel C, Crassous J, et al. Ethylenedithio- tetrathiafulvalene-helicenes: Electroactive helical precursors with switchable chiroptical properties. Chem Eur J. 2013;19(39):13160-7.

40. Hu A, Cao G. Hydrogen-bonded helices in 2-aminothiazole derivatives: Generation of chiral crystals from small achiral molecules. Tetrahedron: Asymmetry. 2011;22(12):1332-6.

41. Popova M, Bretz SL, Hartley CS. Visualizing molecular chirality in the organic chemistry laboratory using cholesteric liquid crystals. J Chem Educ. 2016;93(6):1096-9.

42. Yoshioka S, Inokuma $Y$, Hoshino M, Sato T, Fujita M. Absolute structure determination of compounds with axial and planar chirality using the crystalline sponge method. Chem Sci. 2015;6(7):3765-8.

43. Arroyo-Carmona RE, Reyes-Lucas P, Ramírez-Gutiérrez R, Rivera-Ortega J, Meléndez-Bustamante F, Perez-Benitez A, et al. Teaching helical chirality with a paper helicopter. Chemical Education Journal (CEJ) [serial on the Internet]. 2015;17:10. Available from: http://www.edu.utsunomiya-u.ac.jp/ chem/v17n1/102Perez/perez.html 
PICTORIAL ABSTRACT

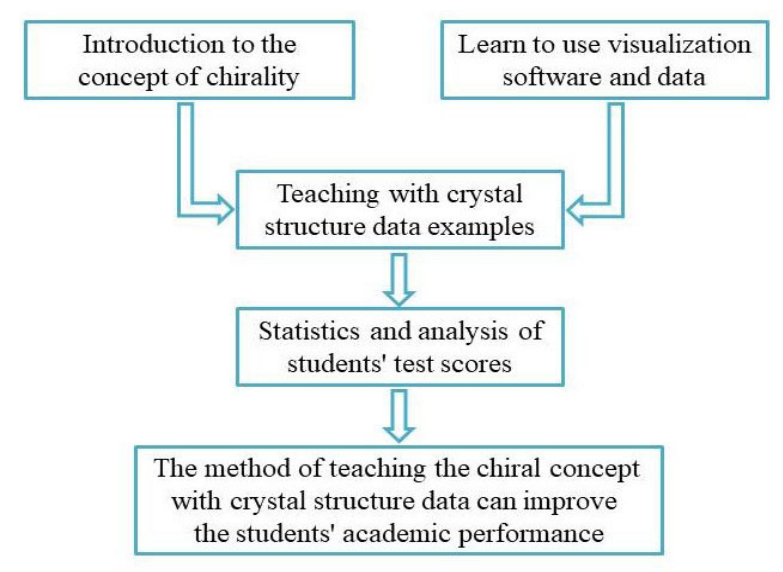

\section{SUMMARY}

The purpose of this study is to introduce a method for teaching the concept of chirality using crystal structure data and 3D Visualization Software. We present many examples and a problem set used in class and have applied this teaching method to students for several years. The students' classroom tests are better than before. The results show that the method of teaching the chiral concept with crystal structure data can improve the students' academic performance.

\section{About Authors}

Ms. Yi Yang, Guangdong Pharmaceutical University, Guangzhou, China. Her current research interests are pharmaceutical chemistry and cosmetics.

Mr. Jian Xu, Guangdong Pharmaceutical University, Guangzhou, China. His main research interest is organic chemistry.

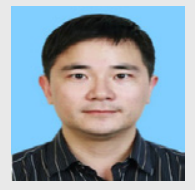

Dr. Gao Cao, Guangdong Pharmaceutical University, Guangzhou, China. He has 11 years of teaching experience, and his current research interests are the synthesis and activity of organic small molecules, the formulation and evaluation of fine chemicals and cosmetics.

Cite this article: Yang Y, Xu J, Cao G. An In-depth Study of the Concept of Chirality in Organic and Pharmaceutical Chemistry: Using Crystal Structure Data with 3D Visualization Software. Indian J of Pharmaceutical Education and Research. 2020;54(4):1133-43. 